\title{
AVALIAÇÃO DO NÍVEL DE CONHECIMENTO SOBRE BOAS PRÁTICAS DE FABRICAÇÃO POR MANIPULADORES DE ALIMENTOS QUE ATUAM EM UNIDADES DE ALIMENTAÇÃO E NUTRIÇÃO
}

\section{EVALUATION OF KNOWLEDGE LEVEL ABOUT GOOD MANUFACTURING PRACTICES BY FOOD MANIPULATORS ACTING IN FOOD AND NUTRITION UNITS}

\author{
Rebeca Emiliano da Silva Solera ${ }^{1}$, Maria Cristina de Almeida Gaspar ${ }^{2}$, Valdirene F. \\ Neves dos Santos ${ }^{3}$ \\ ${ }^{1}$ Universidade Paulista - Instituto de Ciências da Saúde - Rua Antonio Macedo, 505 - CEP \\ 03087 - 010 Parque São Jorge - SP - Brasil. \\ *Autor correspondente: Valdirene Francisca Neves dos Santos. E-mail:<val_usp@hotmail. \\ com> Telefone:011-98596-8855.
}

\begin{abstract}
RESUMO
Nos últimos anos, o consumo de alimentos produzidos em unidades de alimentação e nutrição vem aumentando devido às dificuldades impostas aos trabalhadores, como grandes deslocamentos e extensa jornada de trabalho nas metrópoles brasileiras. Isso aumenta os riscos de aparecimento de doenças transmitidas por alimentos e reforça a necessidade de aplicação das boas práticas de fabricação. Dessa forma, o presente trabalho tem como objetivo avaliar o nível de conhecimento sobre boas práticas de fabricação por manipuladores de alimentos que atuam em unidades de alimentação e nutrição, em estabelecimentos que possuem ou não nutricionista. Foram realizadas pesquisa de campo e observação direta durante o período de dezembro de 2016 a janeiro de 2017 junto aos funcionários de oito unidades de alimentação e nutrição que servem refeições principais na cidade de São Paulo. O questionário foi elaborado com perguntas relacionadas a conhecimentos específicos sobre manipulação de alimentos e variáveis sociodemográficas. Com base nos questionários, o presente estudo demonstra que não houve diferença estatisticamente significante entre os estabelecimentos com e sem nutricionista, pois ambos revelam déficit de conhecimento sobre boas práticas de fabricação por parte dos manipuladores de alimento.
\end{abstract}

Palavras-chave: Doenças Transmitidas por Alimentos; Boas Práticas de Fabricação; Manipulação de Alimentos.

\section{ABSTRACT}

In recent years the consumption of food produced in food and nutrition units has been increasing due to the difficulties imposed on workers by large displacements and long working hours in Brazilian metropolises. This increases the risks of food-borne disease and reinforces the need for applying good manufacturing practices. Thus, the present study aims to evaluate the level of knowledge about good manufacturing practices by food handlers who work in food and nutrition units at establishments that do or do not have a nutritionist. Field research and direct observation during the period from December 2016 to January 2017 was carried out with employees of 8 food and nutrition units serving main meals in the city of São Paulo. The questionnaire was elaborated with 13 questions related to specific knowledge about food manipulation, education level, age and sex. The present study demonstrates that there was no statistically significant difference regarding the questionnaires between establishments with or without a nutritionist, since both revealed deficits in food handlers' knowledge on good manufacturing practices.

Key-Words: Food-borne Diseases; Good Manufacturing Practices; Food Handling. 


\section{INTRODUÇÃO}

Nos últimos anos, o consumo de alimentos produzidos em unidades de alimentação e nutrição vem aumentando devido às dificuldades impostas aos trabalhadores, como grandes deslocamentos e a extensa jornada de trabalho nas metrópoles brasileiras, que levam um grande número de pessoas a realizar suas refeições regulares longe de suas residências(SANTOS; ALVES, 2014).

Com o aumento da demanda nas unidades de alimentação e nutrição, a incidência de doenças relacionadas ao consumo de alimentos cresce anualmente (PASSOS et al.,2008). A maioria dos casos de doenças transmitidas por alimentos não é notificada, pois muitos organismos patogênicos presentes nos alimentos causam sintomas brandos, fazendo com que a vítima não busque auxílio médico (COSTALUNGA; TONDO, 2002).

Grande parte dos surtos alimentares resulta da associação entre o consumo de alimentos contaminados devido a manipulação inadequada e a conservação ou distribuição em condições impróprias (GREIG; RAVEL, 2009).

O manipulador de alimento deve ser instruído por profissionais capacitados, que o sensibilizem sobre os riscos de contaminação e as maneiras adequadas de preparo das refeições, além de instruí-lo sobre as boas práticas de fabricação (BPF) - cujo objetivo é auxiliar os manipuladores no cuidado quanto ao preparo dos alimentos, garantindo a segurança, a qualidade de consumo e a redução das ocorrências das doenças transmitidas por alimentos (BRASIL,2004).

Os manipuladores devem ser instruídos sobre condições higiênico-sanitários dos edifícios; manutenção e higienização das instalações, dos equipamentos e dos utensílios; controle da água de abastecimento; controle integrado de vetores e pragas urbanas; capacitação profissional; controle da higiene e saúde dos manipuladores; manejo de resíduos; e controle e garantia de qualidade do alimento preparado. Esse conhecimento favorece parâmetros adequados de uma alimentação segura (BRASIL,2004).

No entanto, nem sempre as orientações e recomendações dos nutricionistas são aceitas pelos manipuladores dentro de uma unidade de alimentação e nutrição. Em geral, há grande resistência em função da cultura e da experiência de vida de cada manipulador.
Este é o maior desafio no treinamento de boas práticas de fabricação, pois a barreira criada pelo hábito é difícil de ser rompida. Ainda assim, a conscientização dos manipuladores sobre seu papel na prevenção das doenças transmitidas por alimentos é um importante passo (BALARDINI; GUECHESKI; JITKOSKI, 2004).

Dessa forma, o presente trabalho tem como objetivo avaliar o nível de conhecimento sobre boas práticas de fabricação por manipuladores de alimentos que atuam em unidades de alimentação e nutrição em estabelecimentos que possuem ou não nutricionista.

\section{MÉTODOS}

Trata-se de um estudo descritivo, de natureza transversal, numa perspectiva comparativa. Foram realizadas pesquisa de campo e observação direta durante o período de dezembro de 2016 a janeiro de 2017 junto aos funcionários de oito unidades de alimentação e nutrição que servem refeições principais na cidade de São Paulo.

O projeto foi aprovado pelo Comitê de Ética em Pesquisa da Universidade Paulista (UNIP), sob o parecer número 1.838.243. Após a aprovação, foram coletados os dados somente dos funcionários e proprietários que assinaram o Termo de Consentimento Livre e Esclarecido (TCLE), totalizando 115 manipuladores.

No dia da coleta de dados, foram explicados aos manipuladores o objetivo e importância da realização do estudo em questão, assim como foram dadas as instruções de preenchimento dos questionários utilizados.

Como critérios de inclusão, foram considerados manipuladores de alimentos com idade superior a 18 anos, contratados por unidades de alimentação, que exercem o cargo de manipulador de alimento direto ou indireto. A referida pesquisa não contemplou manipuladores de alimentos analfabetos ou menores de 18 anos.

O questionário elaborado foi adaptado de Praxedes (2003) e Guimarães (2006), composto por 13 questões relacionadas a conhecimentos específicos sobre manipulação de alimentos, grau de instrução, idade e sexo.

As respostas corretas foram utilizadas como parâmetros para a análise dos questionários, tendo sido verificado o percentual de acertos. Esses resultados deram subsídios para a efetivação de uma análise comparativa, com o objetivo de averiguar a discrepância entre locais que têm a presença do profissional nutricionista 
e os aqueles que não dispõem desse profissional em relação à qualidade.

Os resultados foram tabulados utilizando o programa Microsoft Office Excel 2013 e, para apresentação dos resultados, foi utilizada estatística descritiva expressa em frequências relativas e absoluta. Posteriormente, os mesmos foram apresentados na forma de gráficos e tabelas.

\section{RESULTADOS E DISCUSSÃO}

Ao todo, 115 manipuladores de alimentos participaram da pesquisa. Nos estabelecimentos com nutricionista, foram avaliados 63 manipuladores, sendo 36 homens e 27 mulheres; e, nos estabelecimentos que não possuem nutricionista, foram avaliados 52 manipuladores, sendo 34 homens e 18 mulheres.

Dos 63 manipuladores participantes da pesquisa que trabalham em estabelecimentos que possuem nutricionista, 53 já haviam participado de algum treinamento de boas práticas de fabricação. Já nos estabelecimentos que não contam com nutricionista, 51 manipuladores já haviam participado de algum treinamento, conforme o gráfico 1.

Gráfico 1 - Número de manipuladores de estabelecimentos com e sem nutricionista que participaram de treinamento de BPF. São Paulo, 2016-2017.

- Treinamento de BPF,Com Nutricionista

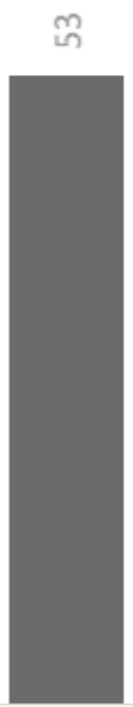

PARTICIPARAM

\section{Treinamento de BPF, Sem Nutricionista}

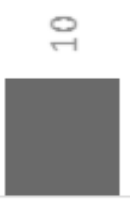

NUNCA PARTICIPARAM

Fundamental Completo e 13\% (n=7), o Ensino Médio Completo, conforme gráfico 2. 
Gráfico 2 - Escolaridade de manipuladores de alimentos de estabelecimentos com e sem nutricionista. São Paulo, 2016 - 2017.

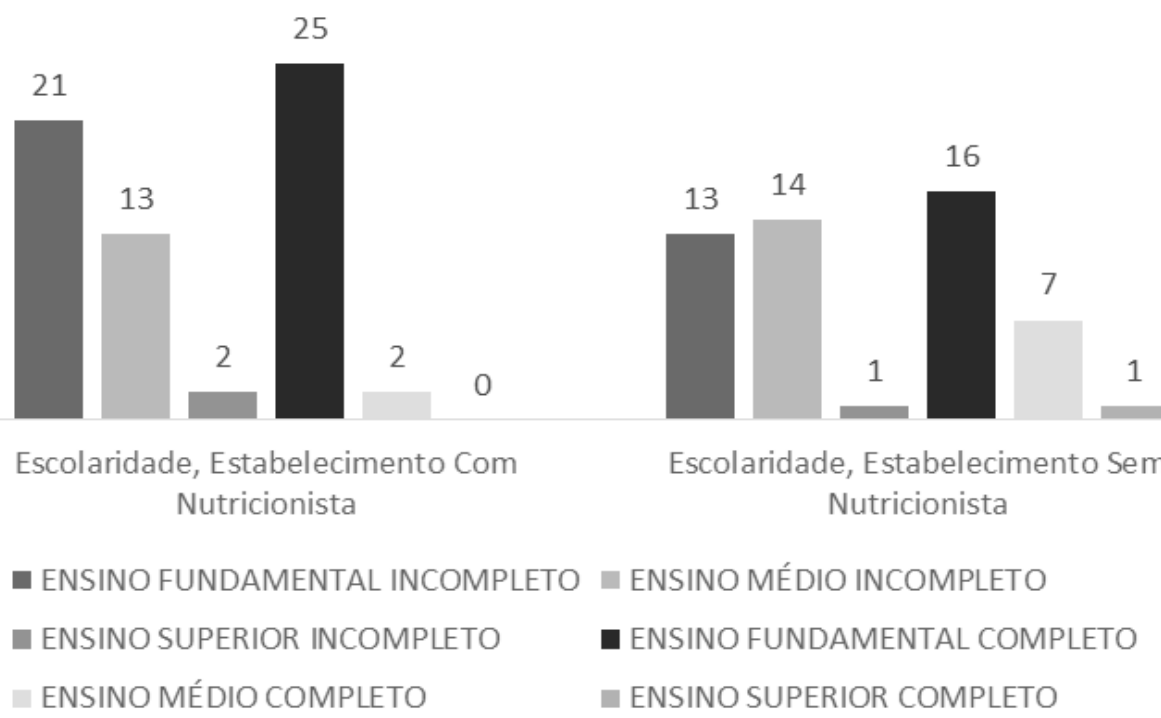

Pesquisas também apontam um baixo nível de escolaridade dos manipuladores de alimentos (PRAXEDES, 2003. SILVA; GERMANO; GERMANO,2003. SOUZA; GERMANO; GERMANO, 2004). Pesquisa com indivíduos responsáveis pela merenda escolar de 24 unidades de ensino fundamental localizadas em São Paulo (SP) revelou que, dos manipuladores de alimentos, apenas $12,5 \%$ haviam concluído o ensino médio (SILVA; GERMANO; GERMANO, 2003). Praxedes (2003) também observou que apenas $18,5 \%$ dos comerciantes ambulantes de alimentos possuíam o ensino médio completo. Dados semelhantes foram encontrados, confirmando a baixa escolaridade entre os manipuladores de alimentos (SOUZA; GERMANO; GERMANO, 2004).

Diante dessa realidade, a capacitação contínua dos manipuladores de alimentos é muito importante, tendo em vista que o setor de alimentação contrata profissionais sem experiência na função a ser desempenhada (MELLO et al., 2010).

Com relação às respostas do questionário de boas práticas de fabricação, foram atribuídos erros e acertos. As porcentagens que não aparecem na tabela 1 correspondem às questões não respondidas ou com respostas inválidas.

Tabela 1. Análise e comparação do conhecimento sobre Boas Práticas de Fabricação.

\begin{tabular}{|c|c|c|}
\hline & $\begin{array}{l}\text { Estabelecimento com } \\
\text { Nutricionista }\end{array}$ & $\begin{array}{l}\text { Estabelecimento sem } \\
\text { Nutricionista }\end{array}$ \\
\hline QUESTÕES & Acertos \%(n) & Acertos \%(n) \\
\hline Você já ouviu falar sobre contaminação de alimentos? & $100 \%(63)$ & $98 \%(51)$ \\
\hline $\begin{array}{l}\text { Quais das seguintes superfícies podem contaminar suas mãos } \\
\text { com microrganismos que podem ser transmitidos aos alimentos? }\end{array}$ & $98 \%(62)$ & $94 \%(49)$ \\
\hline $\begin{array}{l}\text { Você acha que os alimentos contaminados podem desencadear } \\
\text { uma diarreia ou vomito? }\end{array}$ & $78 \%(49)$ & $100 \%(52)$ \\
\hline $\begin{array}{l}\text { Você acha importante ter uma boa higiene pessoal para } \\
\text { trabalhar com os alimentos? }\end{array}$ & $98 \%(62)$ & $100 \%(52)$ \\
\hline Com qual frequência devem ser higienizados os utensílios & $98 \%(62)$ & $100 \%(52)$ \\
\hline O que é considerado falta de higiene? & $98 \%(62)$ & $100 \%(52)$ \\
\hline Quais são as consequências da falta de higiene? & $79 \%(50)$ & $100 \%(52)$ \\
\hline
\end{tabular}


O presente estudo observou que, nos estabelecimentos acompanhados por nutricionista, não houve nenhuma questão com $100 \%$ de acerto. Nos estabelecimentos que não possuem nutricionista, cinco questões tiveram $100 \%$ de acerto.

O treinamento de boas práticas de fabricação deve ser realizado com o objetivo de auxiliar os manipuladores no cuidado quanto ao preparo dos alimentos, garantindo a segurança, a qualidade de consumo e a redução das ocorrências das doenças transmitidas por alimentos. $\mathrm{O}$ conhecimento adquirido no treinamento deve ser colocado em prática no dia a dia, pois, se os manipuladores estiverem em boas condições de saúde e bem capacitados quanto aos procedimentos sanitários, a qualidade dos alimentos servidos à população será melhor, o que irá minimizar o risco de doenças transmitidas por alimentos (CUNHA; AMICHI, 2014)

Em estudo semelhante realizado por Esperança e Marchioni (2011), 22 restaurantes foram analisados: 18 self service e quatro à la carte. O critério utilizado para seleção dos restaurantes foi não ter nenhum responsável técnico como nutricionista, biomédico ou veterinário. Os resultados mostraram que a maior parte dos restaurantes foi classificada como deficiente ou regular. $\mathrm{O}$ item que obteve menor acerto foi boas práticas de fabricação $(9,2 \%)$, e o com o maior acerto foi controle de pragas (71\%). Com esse estudo, pode-se perceber que a qualidade dos serviços oferecidos pelos restaurantes avaliados é deficiente.

Em relação ao conhecimento sobre doenças transmitidas por alimentos, Mello et al. (2010) avaliaram restaurantes públicos populares no estado do Rio de Janeiro e relataram que $69 \%$ dos funcionários responderam incorretamente; destes, $49,5 \%$ haviam sido capacitados. Os manipuladores confundem os tipos de doenças transmitidas por alimentos com sintomas gerais, como colocado a seguir: "Diarreia e qualquer outro tipo de doença"; "Dor no corpo, febre, vômito e diarreia" e "Dor no estômago, de cabeça e diarreia"

Resultado semelhante foi encontrado no trabalho realizado por Silva e Germano (2003) com pessoas responsáveis pela merenda escolar de 24 unidades de ensino fundamental localizadas em São Paulo (SP): $62,5 \%$ afirmaram não conhecer as doenças transmitidas por alimentos, sendo que, destes, $66,7 \%$ haviam sido capacitados.

As questões abordadas no presente estudo são relativas às ações de boas práticas de fabricação presentes no dia a dia dos manipuladores, consideradas de grande importância dentro dos estabelecimentos por se tratar de questões básicas que o manipulador deve conhecer e, portanto, abordadas em treinamentos de boas práticas de fabricação.

Pode-se observar que $100 \%(n=63)$ dos manipuladores acompanhados por nutricionista já ouviram falar sobre contaminação dos alimentos e que $98 \%$ $(\mathrm{n}=51)$ dos manipuladores não acompanhados por nutricionista também já ouviram falar sobre contaminação dos alimentos.

O estudo de Stefanello, Linn e Mesquita (2009) cita que a importância da capacitação é dar aos manipuladores conhecimentos técnico-práticos necessários ao desenvolvimento de habilidades e atitudes de trabalho específicas à área de alimentos. Um treinamento bem elaborado e passado aos manipuladores de forma adequada pode garantir um bom entendimento e, por consequência, uma boa adesão às boas práticas de fabricação.

Diversos fatores podem influenciar a eficácia da formação prática e do conhecimento dos manipuladores de alimentos, tais como: a intervenção/estratégia educacional utilizada; o lugar onde o treinamento é realizado; comparecimento voluntário, ao invés de obrigatório; formação e cultura de trabalho afetadas por normas, 'crenças' e percepções de colegas dos manipuladores de alimentos (CUNHA; STEDEFELDT; ROSSO, 2014).

Além de treinamento, outras características do manipulador de alimentos - como idade, nível de educação e experiência de trabalho - também podem afetar os escores de conhecimento (PICHLER et al., 2014).

Os programas de treinamento são importantes para melhorar o conhecimento de manipuladores de alimentos, porém a melhoria no conhecimento nem sempre resulta em mudanças positivas no comportamento desses trabalhadores. Isso porque, muitas vezes, a adoção de boas práticas é dificultada pelas condições de trabalho, por exemplo: rotinas padronizadas, rígidas e repletas de exigências a serem cumpridas; movimentos repetitivos; levantamento excessivo de peso; permanência em pé por períodos prolongados, agravados por ambientes ruidosos, com temperatura elevada e falta de materiais (FERREIRA et al., 2013). 


\section{CONCLUSÃO}

O presente estudo demonstra que não houve diferença estatisticamente significante referente aos questionários entre os estabelecimentos com e sem nutricionista, porém ambos revelam déficit nos conhecimentos dos manipuladores de alimento sobre boas práticas de fabricação.

Para que ocorram os cuidados descritos para as boas práticas de fabricação, novas estratégias devem ser pensadas pelos nutricionistas e responsáveis pelos estabelecimentos de refeições.

\section{REFERÊNCIAS}

AKUTSU RC. et al. Adequação das boas práticas de fabricação em serviços de alimentação. Rev. Nutr, Campinas, v18, n.3, 2005.

BALARDINI JL, GUECHESKI ARG, JITKOSKI KZ. A Higiene Pessoal do Manipulador na Fabricação de Alimentos. 2004. Disponível em: http://www.unibem.br/ cursos/nutricao/Kath/2.doc. Acesso em 22 de Setembro de 2016.

BRASIL. Ministério da Saúde. Agência Nacional de Vigilância Sanitária. Cartilha sobre Boas Práticas para Serviços de Alimentação. Resolução-RDC n ${ }^{0}$ 216/2004. Brasília ,3ed, 2004.

BRASIL. Ministério da Saúde. Agência Nacional de Vigilância Sanitária. Legislação. Resoluções. Resolução RDC 216 de 15 de Setembro de 2004. Dispõe sobre o regulamento técnico de Boas Práticas para serviços de alimentação. Disponível em: http://e-legis.bvs.br/leisref/ public/showAct.php?id=12546. Acesso em 23 de Agosto de 2016.

COStalunga S, TONDO EC. Salmonellosis in Rio Grande do Sul, Brazil, 1997 to 1999. Brazilian Journal of Microbiology, Brazil, 33ed, p. 342-346, 2002.

CUNHA DT, STEDEFELDT E, ROSSO VV. The role of theoretical food safety training on Brazilian food handlers' knowledge, attitude and practice. Food Control. 43ed, p. 167-174, 2014.

CUNHA L, AMICHI K. Relação Entre a Ocorrência de Enteroparasitoses e Práticas de Higiene de Manipuladores de Alimentos: Revisão da Literatura. Saúde e Pesquisa, América do Norte, v7, n.1, p. 147$157,2014$.

ESPERANÇA LC, MARCHIONI DML. Qualidade na produção de refeições em restaurantes comerciais na região de Cerqueira César, São Paulo. Revista Nutrire, v36, n.1, p. 71-83,2011.
FERREIRA JS. et al. Conhecimento, atitudes e práticas em segurança alimentar de manipuladores de alimentos em hospitais públicos de Salvador, Bahia. Revista Baiana de Saúde Pública, v37, n.1, p. 35-55, 2013.

GREIG JD, RAVEL A. Analysis of foodborne outbreak data reported internationally for source attribution, International Journal of Food Microbiology. 130ed, p. 7787,2009 .

GUIMARÃES KAS. Ações educativas para a promoção da saúde e da segurança do trabalho em restaurantes comerciais. Dissertação (Mestrado em Biociências e Saúde) - Fundação Oswaldo Cruz, Rio de Janeiro, p. 190,2006.

MELlO AG. et al. Conhecimento dos manipuladores de alimentos sobre boas práticas nos restaurantes públicos populares do Estado do Rio de Janeiro. Campinas, v13, n.1, p. 60-68,2010.

PASSOS EC. et al. Surto de toxinfecção alimentar em funcionários de uma empreiteira da construção civil no município de Cubatão, São Paulo/ Brasil. Revista Instituto Adolfo Lutz, v67, n.3, p. 237-240, 2008.

PICHLER J. et al. Evaluating levels of knowledge on food safety among food handlers from restaurants and various catering businesses in Vienna, Austria 2011/2012. Food Control, v35, n.1, p. 33-40, 2014.

PRAXEDES PCG. Aspectos da qualidade higiênicosanitária de alimentos consumidos e comercializados na cidade de São Remo. Dissertação (Mestrado em Epidemiologia Experimental e Aplicada ao Controle das Zoonoses) - Universidade de São Paulo, São Paulo, p. 120, 2003.

SANTOS VFN, ALVES MAA. Unidades de alimentação e nutrição no Brasil: conhecendo o perfil de seus pesquisadores. Linkania Revista Cientifica. 1ed, p. 84 $-185,2014$.

SILVA C, GERMANO MIS, GERMANO PML. Condições higiênico-sanitárias dos locais de preparação da merenda escolar, da rede estadual de ensino em São Paulo/SP. Revista Higiene Alimentar, São Paulo, v17, n.110, p. 4955,2003 .

SOUZA RR, GERMANO PML, GERMANO MIS. Técnica da simulação aplicada ao treinamento de manipuladores de alimentos, como recurso para a segurança alimentar de refeições transportadas. Revista Higiene Alimentar, São Paulo, v18, n.122, p. 21-25,2004.

STEFANELLO CL, LINN DS, MESQUITA MO. Percepção Sobre Boas Práticas por Cozinheiras e Auxiliares de Cozinha de uma UAN do Noroeste do Rio Grande do Sul. Vivências: Revista Eletrônica de Extensão da URI, v5, n. 8 , p. 92-98,2009. 